\title{
Factors associated with positive blood cultures in outpatients with suspected bacteremia
}

\author{
K. Wildi • S. Tschudin-Sutter $\cdot$ S. Dell-Kuster $\cdot$ R. Frei $•$ \\ H. C. Bucher • R. Nüesch
}

Received: 20 September 2010 / Accepted: 5 April 2011 /Published online: 20 April 2011

(C) Springer-Verlag 2011

\begin{abstract}
Blood cultures are routinely taken in outpatients with fever and suspected bacterial infections. However, in the majority of cases, they are not informative and of limited value for clinical decision making. The aim of this study was therefore to investigate factors associated with positive blood cultures in outpatients presenting to an outpatient clinic and emergency room. This was a casecontrol study of all outpatients with positive blood cultures from January 1, 2006 to October 31, 2007 and matched control patients with negative blood cultures in the same time period. Microbiology results and medical charts were
\end{abstract}

Karin Wildi and Sarah Tschudin contributed equally to this work.

K. Wildi · R. Nüesch

Division of Internal Medicine, University Hospital Basel,

Basel, Switzerland

S. Tschudin-Sutter

Division of Infectious Diseases, Hospital Hygiene,

University Hospital Basel,

Basel, Switzerland

S. Dell-Kuster • H. C. Bucher

Basel Institute for Clinical Epidemiology and Biostatistics,

University Hospital Basel,

Basel, Switzerland

R. Frei

Division of Clinical Microbiology, University Hospital Basel,

Basel, Switzerland

R. Nüesch $(\bowtie)$

Clinic for Infectious Diseases and Hospital Epidemiology,

Hirslanden Klinik St. Anna,

St. Anna Str. 32,

6006 Lucerne, Switzerland

e-mail: reto.nuesch@hirslanden.ch

R. Nüesch

e-mail: reto.nuesch@unibas.ch reviewed to determine factors associated with positive blood cultures. The presence of a systemic inflammation response syndrome (SIRS) (OR 2.7, 95\% Cl 1.0-7.2) and increased C-reactive protein (CRP) (OR 1.1 per $10 \mathrm{mg} / \mathrm{l}$, $95 \% \mathrm{Cl} 1.0-1.2)$ were the most powerful predictive values for the development of positive blood cultures. In positive cases serum albumin was lower $(35 \mathrm{mg} / \mathrm{l}$ versus $39 \mathrm{mg} / \mathrm{l})$ than in controls. SIRS, increasing CRP and low albumin were associated with positive blood cultures in outpatients. With simple clinical assessment and few laboratory tests indicative of infection, it is possible to define a group at higher risk for bacteremia in outpatients.

\section{Introduction}

Incidence of sepsis [1] in patients presenting to emergency departments (ED) remains high [2-5] with mortality rates up to $50 \%$ [6]. For this reason early detection and appropriate antibiotic therapy of suspected bacteremia is important.

Blood cultures are routinely taken during the diagnostic work-up of suspected sepsis despite low sensitivity. They are rarely positive in outpatients [7-14] and do not affect patient management when the infection can be established clinically or by microbiological sampling from other body sites $[15,16]$.

Several studies were conducted on inpatients with suspected sepsis to define factors associated with positive blood cultures and to develop guidelines for their rational use $[6,17-26]$. Data that may guide management of outpatients with fever or suspected sepsis syndrome is lacking. The aim of this case-control study was to identify predictive factors for positive blood cultures in outpatients based on findings from clinical examination and routine laboratory testing. 


\section{Methods}

Setting

The University Hospital of Basel is a 680-bed facility with 27,000 inpatients and 167,000 outpatients per year. It provides primary and tertiary care services.

Cases and controls

We evaluated all blood cultures entered into the electronic database of the microbiology laboratory of the University Hospital from January 1, 2006 to October 31, 2007 from patients aged 16 years or older who received ambulatory care or were admitted for less than 48 hours.

The number of blood cultures, the kind of pathogen detected and it's resistance testing, as well as all microbiology culture results from other body samples were extracted from the electronic database in patients with positive blood cultures (cases). White blood cell count, albumin and C-reactive protein (CRP) were collected by chart review, as well as the clinical parameters such as body temperature, heart rate, respiration rate, $\mathrm{PaCO}_{2}$, and comorbidities with a higher risk for bacterial infections including immunosuppression, obstructive lung disease, insulin dependent diabetes, active malignant tumor and liver cirrhosis. Furthermore, data on antibiotic therapy were obtained. We randomly selected patients with negative blood cultures from the same study period as controls and collected the same data. Controls were matched to cases in a 3 to 1 ratio. Two infectious disease specialists independently revaluated all positive blood cultures. Cultures were classified as contaminated if typical skin flora was detected in the minority of blood cultures and another focus of infection could be identified. Discrepant judgement was resolved by consensus. In both groups we evaluated if there was a change in patient management due to the result obtained by blood cultures. Missing information was collected from the referring physician or family practitioner with a standardized letter.

\section{Statistical analysis}

We used the number of blood cultures as the selection criterion for suitable controls for each case. The frequency matching generated equal distributions for the number of blood cultures in cases and controls. To avoid bias of the estimated odds ratios, we included the matching factor as a covariate in the (unconditional) logistic regression model for positive blood cultures. In addition, further covariates were included (age, albumin, CRP, SIRS, presence of co-morbidities) in order to adjust for their possible confounding effect. Patients with missing data of vital signs were considered as not having SIRS. Analysis was made with Intercooled Stata Version 9.2 for Macintosh.

\section{Results}

During the 22-month observation period, 1,432 outpatients and inpatients with less than 48 hours stay had blood cultures taken. Ninety-one patients had positive blood cultures. Of these, 16 were interpreted as contamination, 23 had to be excluded because they were admitted from or went to another institution, and six had died within 48 hours, while data was missing in eight. This resulted in 38 cases with true positive blood cultures, a hospitalisation length $\leq 48$ hours and a well-documented medical history. These cases were matched to 114 blood culture negative controls. In $66 \%$ of all patients (cases and controls), two sets of blood cultures were taken.

Parameters of interest differed between cases and controls (Table 1). Compared to controls, cases were older ( 58 vs. 42 years), were more likely to have SIRS ( $74 \%$ vs. $55 \%$ ), had more underlying diseases and had a lower body temperature $\left(38.5^{\circ} \mathrm{C}\right.$ vs. $\left.39.1^{\circ} \mathrm{C}\right)$. CRP was higher in cases compared to controls $(70 \mathrm{mg} / \mathrm{l}$, vs. $25 \mathrm{mg} / \mathrm{l})$, mean albumin was lower $(35 \mathrm{mg} / \mathrm{l}$ vs. $39 \mathrm{mg} / \mathrm{l})$ and white cell count was higher $(11.2 / \mu \mathrm{l}$ vs. $9.2 / \mu \mathrm{l})$.

In positive cases, commonly found pathogens were $E$. coli $(17 / 38,45 \%)$ and Staphylococcus aureus $(5 / 38,13 \%)$. Polybacterial growth was detected in $7 / 38$ (18.5\%) cases. The detected pathogens were Pseudomonas spp and Clostridium spp. in a patient with diverticulitis, and Enterococcus faecium and Proteus mirabilis in a case of pyelonephritis; also found was bacteremia with $E$. coli and Streptococcus pneumoniae, urosepsis with detection of Acinetobacter spp. and Peptostreptococcus asacharolyticus, an intraabdominal abscess with Klebsiella pneumoniae and E.coli, bacteremia with $S$. aureus and viridans Streptococci, as well as bacteremia with detection of Fusobacterium necrophorum and Bacteroides ureolyticus. Frequent infections were urinary tract infection $(39.9 \%)$, fever without focus (21\%), pneumonia (7.9\%), sepsis (5.3\%), endocarditis (5.3\%), unspecified infection (5.3\%), and diverticulitis $(2.2 \%)$. The most frequent infections in controls were fever without focus $(30 \%)$, pneumonia $(25 \%)$, viral infection $(23 \%)$, soft tissue infection (5.3\%), urinary tract infection (7.8\%), and otolaryngological infections (3.5\%). A total of 23 cases $(61 \%)$ received initial antibiotic treatment, mostly with quinolones (12/38) or an aminopenicillin (6/38) and 45 control patients $(40 \%)$ received antibiotic therapy with amoxicillin/clavulanate. In 21 cases (55\%) cultures from other body sites had been taken (mostly urine cultures) and in 16 patients $(76 \%)$ bacteria were detected in these cultures. In $14(88 \%)$, identical isolates were found in the blood cultures.

In uni- and multivariate logistic regression analysis factors associated with positive blood cultures were the presence of SIRS (OR 2.7; 95\%CI 2.0-7.2), serum albumin (OR per $10 \mathrm{mg}$ decrease $0.4 ; 95 \% \mathrm{CI} 0.4-0.9$ ) and CRP (per 
Table 1 Patients characteristics

\begin{tabular}{|c|c|c|c|c|}
\hline Characteristics & Controls $(n=114)$ & Cases $(n=38)$ & Total $(n=152)$ & Missing parameters \\
\hline Age & $42(16-92)$ & $58(18-96)$ & $43(16-96)$ & - \\
\hline \multicolumn{5}{|l|}{ Number of blood cultures } \\
\hline $1 \times 2$ & 18 & 6 & $24(16 \%)$ & \\
\hline $2 \times 2$ & 75 & 25 & $100(66 \%)$ & \\
\hline $3 \times 2$ & 18 & 6 & $24(16 \%)$ & \\
\hline $4 \times 2$ & 3 & 1 & $4(3 \%)$ & \\
\hline Pulse (per minute) & $93(56-147)$ & $104(56-140)$ & $94(56-147)$ & 13 \\
\hline Temperature $\left({ }^{\circ} \mathrm{C}\right)$ & 39.1 ( $37.1-42.3)$ & $38.5(36.1-40.5)$ & $38.5(36.1-42.3)$ & 12 \\
\hline Respiration frequency (per minute) & $16(12-36)$ & $16(12-32)$ & $16(12-36)$ & 105 \\
\hline aBGA: Alkalosis, $n(\%)$ & $2(2 \%)$ & $1(3 \%)$ & $3(2 \%)$ & 140 \\
\hline White cell count $(\mu \mathrm{L})$ & $9.2(0.8-27)$ & $11.2(2.5-26)$ & $9.3(0.8-27)$ & 1 \\
\hline Premature neutrophils & $14(12 \%)$ & $3(8 \%)$ & $17(11 \%)$ & 129 \\
\hline SIRS absent & $23(20 \%)$ & $7(18 \%)$ & $30(20 \%)$ & \\
\hline SIRS present, $n(\%)$ & $63(55 \%)$ & $28(74 \%)$ & $91(60 \%)$ & \\
\hline SIRS not defined, $n(\%)$ & $28(25 \%)$ & $3(8 \%)$ & $31(20 \%)$ & \\
\hline CRP (mg/l) & $25(1-239)$ & $70(3-255)$ & $35(1-255)$ & 1 \\
\hline Albumin (mg/l) & $39(22-50)$ & $35(16-47)$ & $37(16-50)$ & 3 \\
\hline \multicolumn{5}{|l|}{ Comorbidities } \\
\hline Immunodepression, $n(\%)$ & $22(19 \%)$ & $11(29 \%)$ & $33(22 \%)$ & \\
\hline $\mathrm{COPD}, n(\%)$ & $14(12 \%)$ & $5(13 \%)$ & $19(13 \%)$ & \\
\hline Insulin dependent diabetes, $n(\%)$ & $2(2 \%)$ & $4(11 \%)$ & $6(4 \%)$ & \\
\hline Active malignoma, $n(\%)$ & $14(12 \%)$ & $6(16 \%)$ & $20(13 \%)$ & \\
\hline Liver cirrhosis, $n(\%)$ & - & $1(3 \%)$ & $1(1 \%)$ & \\
\hline \multicolumn{5}{|l|}{ Number of comorbidities, $n(\%)$} \\
\hline 0 & $82(72 \%)$ & $19(50 \%)$ & $101(66 \%)$ & \\
\hline 1 & $15(13 \%)$ & $11(29 \%)$ & $26(17 \%)$ & \\
\hline 2 & $14(12 \%)$ & $8(21 \%)$ & $22(14 \%)$ & \\
\hline 3 & $3(3 \%)$ & - & $3(2 \%)$ & \\
\hline
\end{tabular}

Noted as median and range, and percentage from the total

$10 \mathrm{mg}$ increase OR 1.1; 95\%CI 1.0-1.2). Increased age and comorbidities were associated with higher odds for positive bloods cultures in uni- but not in multivariate analysis (Table 2).
Data on follow-up in cases was available in 38 patients. In controls, follow-up data was available in 95 of 114 patients. Thirteen cases $(34 \%)$ were re-evaluated following positive results of their blood cultures - six were hospitalised, of

Table 2 Univariate and multivariable logistic regression analysis

\begin{tabular}{|c|c|c|c|c|}
\hline \multirow[t]{2}{*}{ Covariates } & \multicolumn{2}{|l|}{ Unadjusted analysis } & \multicolumn{2}{|l|}{ Adjusted analysis ${ }^{\mathrm{a}}$} \\
\hline & Odds ratio $(95 \% \mathrm{CI})$ & $p$-value & Odds ratio $(95 \% \mathrm{CI})$ & $p$-value \\
\hline Age per 10 years & $1.3(1.0 ; 1.5)$ & 0.02 & $1.2(0.9 ; 1.5)$ & 0.1 \\
\hline Albumin per $10 \mathrm{mg} / 1$ amount & $0.2(0.1 ; 0.5)$ & $<0.001$ & $0.4(0.2 ; 0.8)$ & 0.02 \\
\hline CRP per $10 \mathrm{mg} / \mathrm{l}$ amount & $1.1(1.0 ; 1.2)$ & $<0.001$ & $1.1(1.0 ; 1.2)$ & 0.01 \\
\hline SIRS: criteria fulfilled vs. not fulfilled or no definition & $2.4(1.0 ; 5.5)$ & 0.04 & $2.7(1.0 ; 7.2)$ & 0.04 \\
\hline Comorbidities: yes vs. none & $2.5(1.2 ; 5.5)$ & 0.02 & $1.5(0.6 ; 3.7)$ & 0.4 \\
\hline
\end{tabular}

The number of patients was reduced to 149 because of missing data for albumin and CRP in three patients

${ }^{a}$ Adjustment for the number of blood cultures and all other co-variates 
which four received additional antibiotics and two had a change in antibiotic therapy. Of seven cases with ambulatory management, two patients were newly started on antibiotic therapy and five had their antibiotic therapy changed. In seven patients with change of initial therapy, two had pathogens resistant to the previous antibiotic. In the control group we found no change in patient management stopping or shortening of the antibiotic therapy due to negative results of blood cultures.

\section{Discussion}

In this case control study of outpatients with suspected bacterial infection from a single academic centre in Switzerland, presence of SIRS, increases in CRP and decreases in albumin were factors most likely to be associated with positive blood cultures. However, results of positive blood cultures only affected patient management in one third of cases and in none of the controls, and the overwhelming majority o blood cultures remained negative. Our data further indicates that blood cultures add little information if a culture can be directly taken from the infected site. Findings on the number of positive blood cultures and outpatients undergoing therapeutic consequences from blood culture results are comparable to findings by others [27, 28]. However, our study included a control group and only looked at outpatients, which in comparison to previous studies on this topic, is a strength. We found lower rates of contaminated blood cultures than previous studies $[16,17,22,29-31]$.

Jaimes et al. [25] identified heart beat $\geq 90 / \mathrm{min}$, temperature $\geq 37.8^{\circ} \mathrm{C}$ and white cell count $>12 / \mu l$ as predictors for bacteremia in inpatients. These factors are constituent of the SIRS definition and confirm our findings of increased odds for positive blood cultures in outpatients admitted with SIRS.

Hypoalbuminaemia is known to occur in severe infections [32]; however, to our knowledge no study so far has found an association for hypoalbuminemia and positive blood cultures.

Several studies have found an association between CRP and bacteremia [33-38] in intensive care patients and identified CRP as a marker for confirmed bacteremia [39], or a marker of sepsis in ICU patients with SIRS [40]. The kinetics and the low specificity of CRP are well known and therefore interpretation of CRP in patients with suspected bacterial infection should always be done in context with SIRS symptoms and clinical presentation.

Our study has some limitations. Confidence intervals for the associations found are wide, indicating the relative lack of power and need for careful interpretation. The retrospective design of our study precluded us from collecting additional data for the clinical indication to take blood cultures. Many patients had only one set of blood cultures taken. One blood culture set detects only $90-91.5 \%$ of bacteremia [41-43]. Thus, we may have included patients with false negative results in the control group. Finally, this data is from a single academic centre and needs confirmation in other settings.

In conclusion, this study indicates that the presence of SIRS, elevated levels of CRP and low albumin are associated with positive blood cultures in outpatients presenting with suspected infection to an ED. The identified factors need confirmation in prospective studies but may indicate a promising approach to reduce the number of blood cultures that will not affect patient management but add additional costs.

\section{References}

1. Bone RC, Sibbald WJ, Sprung CL (1992) The ACCP-SCCM consensus conference on sepsis and organ failure. Chest 101:14811483

2. Rangel-Frausto MS, Pittet D, Costigan M, Hwang T, Davis CS, Wenzel RP (1995) The natural history of the systemic inflammatory response syndrome (SIRS). A prospective study. JAMA 273:117-123

3. Martin GS, Mannino DM, Eaton S, Moss (2003) The epidemiology of sepsis in the United States from 1979 through 2000. N Engl J Med 348:1546-1554

4. Sands KE, Bates DW, Lanken PN, Graman PS, Hibberd PL, Kahn KL, Parsonnet J, Panzer R, Orav EJ, Snydman DR, Black E, Schwartz JS, Moore R, Johnson BL Jr, Platt (1997) Epidemiology of sepsis syndrome in 8 academic medical centers. JAMA 278:234-240

5. Engel C, Brunkhorst FM, Bone HG, Brunkhorst R, Gerlach H, Grond S, Gruendling M, Huhle G, Jaschinski U, John S, Mayer K, Oppert M, Olthoff D, Quintel M, Ragaller M, Rossaint R, Stuber F, Weiler N, Welte T, Bogatsch H, Hartog C, Loeffler M, Reinhart (2007) Epidemiology of sepsis in Germany: results from a national prospective multicenter study. Intensive Care Med 33:606-618

6. Bates DW, Cook EF, Goldman L, Lee T (1990) Predicting bacteremia in hospitalized patients. A prospectively validated model. Ann Intern Med 113:495-500

7. Chalasani NP, Valdecanas MA, Gopal AK, McGowan JE Jr, Jurado R (1995) Clinical utility of blood cultures in adult patients with community-acquired pneumonia without defined underlying risks. Chest 108:932-936

8. Campbell SG, Marrie TJ, Anstey R, Ackroyd-Stolarz S, Dickinson G (2003) Utility of blood cultures in the management of adults with community acquired pneumonia discharged from the emergency department. Emerg Med J 20:521-523

9. Corbo J, Friedman B, Bijur P, Gallagher EJ (2004) Limited usefulness of initial blood cultures in community acquired pneumonia. Emerg Med J 21:446-448

10. Theerthakarai R, El-Halees W, Ismail M, Solis RA, Khan MA (2001) Nonvalue of the initial microbiological studies in the management of nonsevere community-acquired pneumonia. Chest 119:181-184

11. Pasternak EL 3rd, Topinka MA (2000) Blood cultures in pyelonephritis: Do results change therapy? Acad Emerg Med $7: 1170$ 
12. Sturmann KM, Bopp J, Molinari D, Akhtar S, Murphy J (1996) Blood cultures in adult patients released from an urban emergency department: a 15-month experience. Acad Emerg Med 3:768-775

13. Perl B, Gottehrer NP, Raveh D, Schlesinger Y, Rudensky B, Yinnon AM (1999) Cost-effectiveness of blood cultures for adult patients with cellulitis. Clin Infect Dis 29:1483-1488

14. Stevenson A, Hider P, Than M (2005) The utility of blood cultures in the management of non-facial cellulitis appears to be low. $\mathrm{N} \mathrm{Z}$ Med J 118:U1351

15. Kelly AM (1998) Clinical impact of blood cultures taken in the emergency department. J Accid Emerg Med 15:254-256

16. Laupland KB, Church DL, Gregson DB (2005) Blood cultures in ambulatory outpatients. BMC Infect Dis 5:35

17. Shapiro NI, Wolfe RE, Wright SB, Moore R, Bates DW (2008) Who needs a blood culture? A prospectively derived and validated prediction rule. J Emerg Med 35:255-264

18. Mozes B, Milatiner D, Block C, Blumstein Z, Halkin H (1993) Inconsistency of a model aimed at predicting bacteremia in hospitalized patients. J Clin Epidemiol 46:1035-1040

19. Leibovici L, Greenshtain S, Cohen O, Mor F, Wysenbeek AJ (1991) Bacteremia in febrile patients. A clinical model for diagnosis. Arch Intern Med 151:1801-1806

20. Mylotte JM, Pisano MA, Ram S, Nakasato S, Rotella D (1995) Validation of a bacteremia prediction model. Infect Control Hosp Epidemiol 16:203-209

21. Yehezkelli Y, Subah S, Elhanan G, Raz R, Porter A, Regev A, Leibovici L (1996) Two rules for early prediction of bacteremia: testing in a university and a community hospital. J Gen Intern Med 11:98-103

22. Salluzzo R, Reilly K (1991) The rational ordering of blood cultures in the emergency department. Qual Assur Util Rev 6:28-31

23. Fontanarosa PB, Kaeberlein FJ, Gerson LW, Thomson RB (1992) Difficulty in predicting bacteremia in elderly emergency patients. Ann Emerg Med 21:842-848

24. Pfitzenmeyer P, Decrey H, Auckenthaler R, Michel JP (1995) Predicting bacteremia in older patients. J Am Geriatr Soc 43:230 235

25. Jaimes F, Arango C, Ruiz G, Cuervo J, Botero J, Velez G, Upegui N, Machado F (2004) Predicting bacteremia at the bedside. Clin Infect Dis 38:357-362

26. Lyman JL (1986) Use of blood cultures in the emergency department. Ann Emerg Med 15:308-311

27. Ehrenstein BP, Jarry T, Linde HJ, Scholmerich J, Gluck T (2005) Low rate of clinical consequences derived from results of blood cultures obtained in an internal medicine emergency department. Infection 33:314-319

28. Mountain D, Bailey PM, O'Brien D, Jelinek GA (2006) Blood cultures ordered in the adult emergency department are rarely useful. Eur J Emerg Med 13:76-79
29. Stalnikowicz R, Block C (2001) The yield of blood cultures in a department of emergency medicine. Eur J Emerg Med 8:93-97

30. Howie N, Gerstenmaier JF, Munro PT (2007) Do peripheral blood cultures taken in the emergency department influence clinical management? Emerg Med J 24:213-214

31. Kamin Y, Steinberg JM, Kafka M, Hussein A, Srugo I (2003) Is there a need for taking blood cultures from febrile adults discharged from the emergency department? J Infect 46:72-73

32. Fleck A, Raines G, Hawker F, Trotter J, Wallace PI, Ledingham IM, Calman KC (1985) Increased vascular permeability: a major cause of hypoalbuminaemia in disease and injury. Lancet 1:781-784

33. Gabay C, Kushner I (1999) Acute-phase proteins and other systemic responses to inflammation. N Engl J Med 340:448-454

34. Povoa P (2002) C-reactive protein: a valuable marker of sepsis. Intensive Care Med 28:235-243

35. Povoa P, Almeida E, Moreira P, Fernandes A, Mealha R, Aragao A, Sabino H (1998) C-reactive protein as an indicator of sepsis. Intensive Care Med 24:1052-1056

36. Miller PR, Munn DD, Meredith JW, Chang MC (1999) Systemic inflammatory response syndrome in the trauma intensive care unit: who is infected? J Trauma 47:1004-1008

37. Hambach L, Eder M, Dammann E, Schrauder A, Sykora KW, Dieterich C, Kirschner P, Novotny J, Ganser A, Hertenstein B (2002) Diagnostic value of procalcitonin serum levels in comparison with C-reactive protein in allogeneic stem cell transplantation. Haematologica 87:643-651

38. Reny JL, Vuagnat A, Ract C, Benoit MO, Safar M, Fagon JY (2002) Diagnosis and follow-up of infections in intensive care patients: value of C-reactive protein compared with other clinical and biological variables. Crit Care Med 30:529-535

39. Chirouze C, Schuhmacher H, Rabaud C, Gil H, Khayat N, Estavoyer JM, May T, Hoen B (2002) Low serum procalcitonin level accurately predicts the absence of bacteremia in adult patients with acute fever. Clin Infect Dis 35:156-161

40. Sierra R, Rello J, Bailen MA, Benitez E, Gordillo A, Leon C, Pedraza S (2004) C-reactive protein used as an early indicator of infection in patients with systemic inflammatory response syndrome. Intensive Care Med 30:2038-2045

41. Lee A, Mirrett S, Reller LB, Weinstein MP (2007) Detection of bloodstream infections in adults: how many blood cultures are needed? J Clin Microbiol 45:3546-3548

42. Weinstein MP (1996) Current blood culture methods and systems: clinical concepts, technology, and interpretation of results. Clin Infect Dis 23:40-46

43. Weinstein MP, Murphy JR, Reller LB, Lichtenstein KA (1983) The clinical significance of positive blood cultures - a comprehensive analysis of 500 episodes of bacteremia and fungemia in adults. 2. Clinical observations, with special reference to factors influencing prognosis. Rev Infect Dis 5:54-70 